\title{
Noninvasive Assessment of Local Myocardium Repolarization Changes using High Resolution Surface ECG Mapping
}

\author{
M. TYŠLER ${ }^{1,2}$, P. KNEPPO ${ }^{1}$, M. TURZOVÁ ${ }^{2}$, J. ŠVEHLÍKOVÁ $^{2}$, S. KARAS ${ }^{2}$, E. \\ HEBLÁKOVÁ ${ }^{2}$, K. HÁNA ${ }^{1}$, S. FILIPOVÁ ${ }^{3}$ \\ ${ }^{1}$ Czech Technical University, Faculty of Biomedical Engineering, Kladno, Czech Republic, \\ ${ }^{2}$ Institute of Measurement Science, Slovak Academy of Sciences, Bratislava, Slovakia, \\ ${ }^{3}$ Slovak Healthcare University, Bratislava, Slovakia
}

Received May 23, 2007

Accepted May 29, 2007

On-line available May 31, 2007

\begin{abstract}
Summary
A method using body surface potential maps for assessment of myocardium lesions with changed repolarization is presented and suitable mapping system is introduced. Differences between normal and altered QRST integral maps together with torso volume conductor model were used to determine the equivalent dipole representing the lesion. Performance of the method was studied on simulated data. Changed repolarization was modeled by shortening of myocyte action potentials in regions typical for stenosis of the main coronary arteries. The equivalent dipole estimated the positions of small lesions with a mean error of $9 \pm 4 \mathrm{~mm}(17 \pm 14 \mathrm{~mm}$ for larger transmural lesions). The subepicardial or subendocardial character of the lesions was reflected in the dipole orientation. Tests of the method on patients after myocardial infarction that underwent coronary intervention on a single coronary vessel showed that in 7 of 8 successfully treated patients the dipole position matched well with the treated vessel. A small dipole moment in another patient indicated unsuccessful treatment. The method was implemented in a new 128-channel mapping system. Its active electrodes, battery powered measuring unit and optical computer interface help to minimize noise in ECG and guarantee patient's safety. The results suggest that the method and mapping system offer useful tools for noninvasive identification of local repolarization changes in the myocardium.
\end{abstract}

Key words

Myocardium repolarization $\bullet$ Equivalent dipole model $\bullet$ Body surface potential mapping $\bullet$ Active ECG electrodes

\section{Introduction}

The depolarization process of heart ventricles is expressed in the QRS complex of the ECG signals and is determined particularly by the spread of the activation through the myocardial cells (by individual times of their depolarization). The repolarization phase and its expression in the ST-T part of the ECG signals is strongly influenced by the form of the action potential (AP) of myocardial cells (Mirvis 1993). The typical AP form has several parts, the principal ones being initial rapid depolarization (increase of AP from resting to maximum 
level), slow repolarization phase (plateau) and a final rapid repolarization phase. At least three types of cells from endo- to epicardium with slightly different AP forms and durations were revealed experimentally (Antzelevitch et al. 1999). Differences between the AP forms in the endo- mid- and epicardial myocardium layers and transmural distribution of AP duration seem to be important for the resulting repolarization process and its projection into surface ECG signals (Antzelevitch 2001).

Ischemia of myocardial cells is connected with their altered repolarization, partiularly with shortening and decrease of their AP (Lukas et al. 1993). These changes are reflected also in altered heart surface electrograms (Abildskov et al. 1980) and body surface ECG potentials and it is generally accepted and confirmed by advanced model studies (Trudel et al. 2004) that integrals of body surface ECG potentials over the whole ventricular depolarization-repolarization period (QRST interval in ECG) practically depend only on the AP forms and not on the ventricular activation sequence (Abildskov et al. 1979). Measured changes in surface QRST integrals thus can be used for assessment of repolarization changes in the myocardium (Gardner et al. 1981).

Body surface potential (BSP) mapping is a noninvasive electrocardiographic method permitting more precise analysis of the cardiac electric field based on detailed registration of surface potentials using a high number of sensing electrodes (de Ambroggi et al. 1989). Forty years of experience with several mapping lead sets showed that information contents in BSP maps obtained from 24 to 240 leads is greater than that of commonly used standard 12-lead ECG or Frank VCG (Lux et al. 1978, Kornreich et al. 1985, Hoekema et al. 1999). Although the measured BSP maps can be used for immediate clinical diagnostics (SippesGroenewegen et al. 1994, Kozlíková et al. 1997), several studies proved that BSP maps together with information on torso volume conductor obtained e.g. from MR, CT or US imaging systems can be used for more advanced studies based on inverse solutions and more or less simplified equivalent cardiac electric generators that permit noninvasive assessment of abnormal electrical sources in the cardiac tissue (Geselowitz 1963, Malmivuo 1985, Gulrajani et al. 1989).

In this study we present a method for noninvasive identification of local ischemic lesions with abnormal myocyte repolarization that can be caused by stenosis of a single coronary vessel. The method determines an equivalent dipole (ED) (Gabor et al. 1954) representing the local electrical sources due to the altered repolarization. Parameters of the dipole model are estimated from differences in surface QRST integrals that are obtained from BSP maps measured in situations with and without manifestation of the ischemia.

We also introduce a newly developed high resolution 128 channel ECG mapping system that can be used for BSPmap-based studies of the cardiac electric field. Results of experimental application of the system for identification of local repolarization changes using 32 surface leads are presented.

\section{Methods}

Cardiac electric potentials on a chest surface of known geometry and internal electrical conductivities, measured under normal conditions of the myocardium and under conditions with locally changed myocyte repolarization were investigated. Changes between surface potentials in these two situations, represented by difference QRST integral maps (DI maps) were used for noninvasive assessment of the myocardium region with changed repolarization.

For a small region, differences in body surface potentials can be interpreted as being caused by a single dipolar source evoked by the AP alternation during the repolarization phase and located at the center of the region. Such a source can be described by the well known moving dipole model (Savard et al. 1980). However, to avoid solving the nonlinear problem of estimating moving dipole parameters and to guarantee localization of the dipole within the heart walls we applied a fixed dipole model located at one of the predefined locations in the myocardium. For each of these locations $i=1,2, \ldots n$, an equivalent dipole source representing the changes in body surface potentials was estimated using the formula:

$D_{i}=T_{i}^{+} \Phi$

where $D_{i} \quad$ is an estimate of the dipole moment of an ED located in the $\mathrm{i}$-th predefined position, $T_{i}^{+}$is pseudoinverse of a transfer matrix, $\Phi$ are differences in QRST integrals of surface potentials in mapped surface points. The dipole moment $D_{i}$ in fact represents the integral of the dipole moment of physical current dipole in the i-th position during the QRST (depolarizationrepolarization) period.

Transfer matrix $T_{i}$ represents the relation 
between the position of the i-th dipole and the surface potentials and reflects only the geometrical and electrical properties of the torso volume conductor. To solve the inverse problem of $D_{i}$ estimation, pseudo-inverse $T_{i}^{+}$was computed using singular value decomposition of $T_{i}$.

The criterion for finding the location of the ED that best represents changes in surface potentials was the minimal rms difference $R_{D}$ between the original DI map (values $\Phi_{\mathrm{n}}$ ) and a map generated by the ED (values $F_{\mathrm{n}}$ ) in the same torso surface points:

$$
R_{D}=\frac{\sqrt{\sum_{n}\left(F_{n}-\phi_{n}\right)^{2}}}{\sqrt{\sum_{n} \phi_{n}^{2}}} \quad \text { for } n=1,2, \ldots L
$$

where $L$ is the number of measured leads (torso surface points). The magnitude of this difference also served as a measure showing to which extent the ED is able to represent the region with changed repolarization. The performance of the method was first analyzed on simulated surface potentials and its ability to identify a modeled region with changed repolarization was studied.

\section{Analysis of the method on simulated data}

A forward model was used to simulate body surface potentials in a normal case and in the case with locally changed repolarization of the ventricles. An analytically defined ventricular model was formed by several ellipsoids (Szathmáry et al. 1998) and up to five layers with different AP characteristics were defined in the ventricular walls and in the septum for repolarization simulation. Realistic AP shapes and durations as measured in canine left ventricular wedge preparation (Yan et al. 1998) were adopted. Transmural dispersion of AP durations was about $40 \mathrm{~ms}$. Similar transmural AP heterogeneity was observed in isolated human myocytes (Li et al. 1998). A cellular automaton was employed to simulate cardiac depolarization-repolarization process.

Ischemic lesions were simulated by shortening the AP duration by up to $20 \%$ from the normal values. Three regions of changed AP, typical for stenosis of the main coronary vessels were defined (Fig. 1.): A - in the antero-septal part of the left ventricle near the apex, supplied by left anterior descending coronary artery, LAD; $\mathrm{P}$ - in the postero-lateral part of the left ventricle (LV) close to the heart base and supplied by circumflex coronary artery, Cx; I - inferior, in mid postero-septal part of the left and right ventricle (LV, RV) supplied by

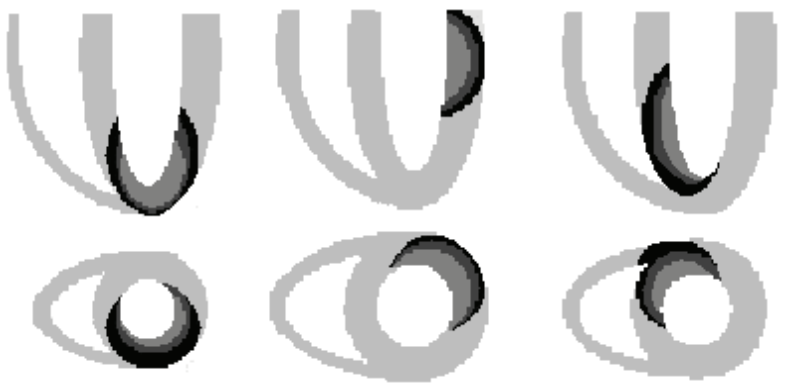

Fig. 1. Simulated subendocardial regions with changed repolarization in the ventricular myocardium model. Three lesion sizes (marked by different grey levels) were simulated in each region. Left: antero-septal regions $\mathrm{A} 1, \mathrm{~A} 2, \mathrm{~A} 3$ in the $\mathrm{LV}$; center: postero-lateral regions $\mathrm{P} 1, \mathrm{P} 2, \mathrm{P} 3$ in the LV; right: inferior regions I1, I2, I3 in the mid postero-septal LV and RV. Cuts through the lesion centers are shown.

the right coronary artery, RCA. In each region, small and medium subendocardial lesions and large transmural lesions (comprising 3-4\%, 6-8\% or 10-12\% of the ventricular volume, respectively) were simulated and marked by numbers 1,2 or 3 . Similarly, small subepicardial lesions (marked as AE, PE and IE) were defined in the same three regions.

For computation of surface potentials in a realistic torso model with lungs and heart cavities, 168 dipoles representing anatomically defined segments of the ventricular model were used (Tyšler et al. 2003, Szathmáry et al. 2002). Body surface potentials were computed in 3 lead sets: in 192 leads of a 16×12 grid (G192), in 62 leads of the Amsterdam mapping set (A62) and in 32 leads of the anterior lead set according to Lux (L32). The second and third lead sets were subsets of the first one.

For inverse estimation of the ED source from simulated data, 298 nodes on the epi- and endocardial ventricular surface were defined as possible ED positions and a dipolar source obtained by summing elementary sources within the region with changed repolarization and placed in its gravity center was considered as correct representation of the lesion.

The same inverse estimations of the ED were computed from surface potentials contaminated by some level of disturbances caused mainly by slow baseline fluctuations. We simulated the influence of these fluctuations as random superpositions with a normal distribution $N\left(0, \sigma^{2}\right)$ and a set the standard deviations $\sigma$ of 1 to $4 \mathrm{mV} . \mathrm{ms}$. Corresponding levels of the disturbances were marked as N1 to N4. The size and position of the region with altered repolarization, number 


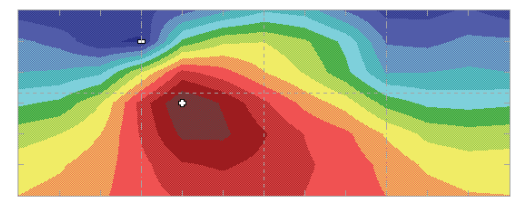

NORMAL

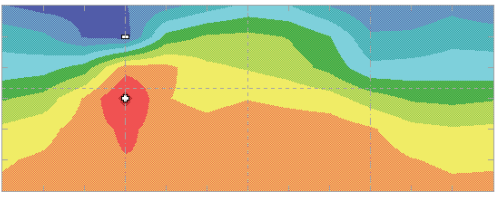

A2

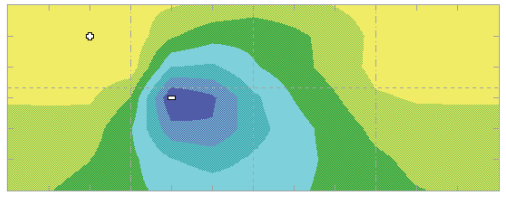

DIFFERENCE A2

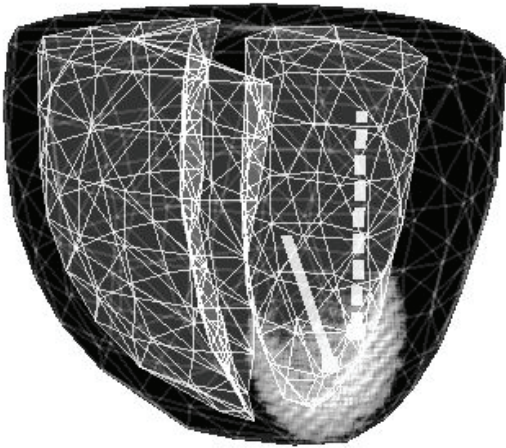

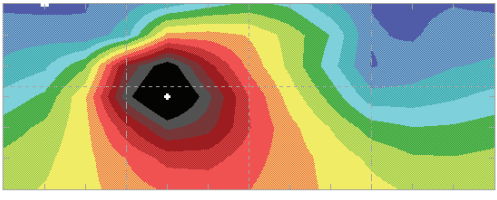

P2

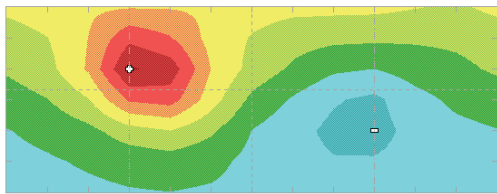

DIFFERENCE P2

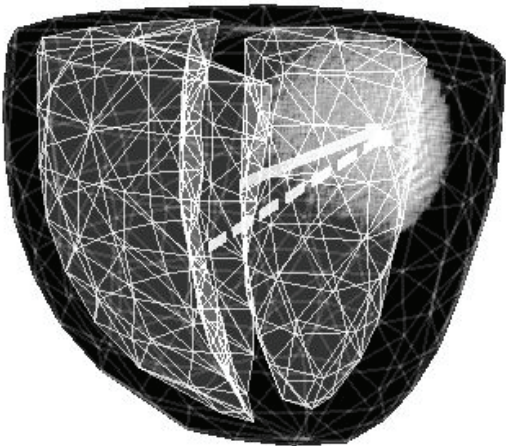

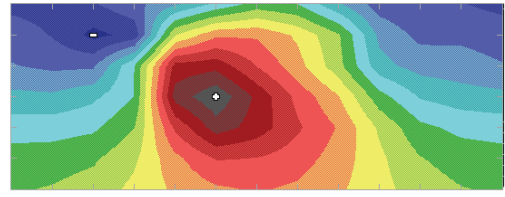

12

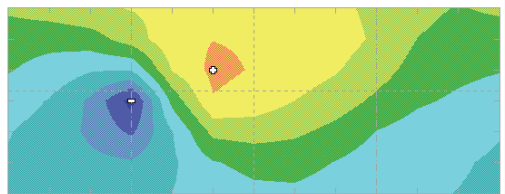

DIFFERENCE 12

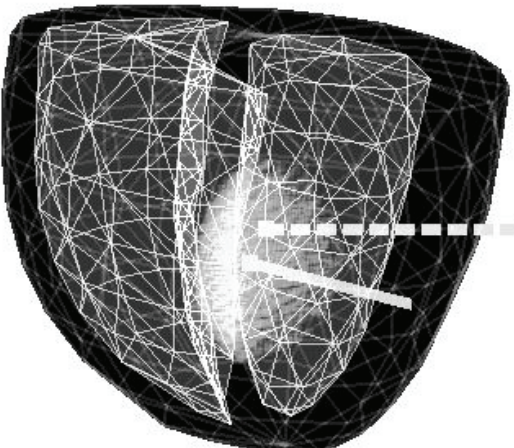

Fig. 2. Top: Simulated QRST integral maps for normal depolarization-repolarization (NORMAL) and for activations with AP shortened by $20 \%$ in lesions of medium size located in antero-septal (A2), postero-lateral (P2) and postero-septal (I2) part of the ventricular myocardium. Corresponding difference QRST integral maps are shown in the bottom row. Step in maps is 6 mV.s. Bottom: Simulated lesions same as above and their dipole representations: left: A2 in the antero-septal wall of the LV; center: P2 in the postero-lateral wall of the LV; right: I2 lesion in the mid postero-septal part of the LV and RV. Original dipole moments computed as sum of simulated elementary dipole moments in the lesion are marked by full lines, inversely estimated dipole moments of the ED inversely calculated from the simulated surface ECG potentials are marked by dashed lines.

of measured surface leads, neglect of inhomogeneities in the torso model used in the inverse computation and possible random disturbances in QRST integrals were analyzed as factors influencing accuracy of the method.

\section{Testing of the method on measured ECG data}

For experimental testing of the method, measured data from 12 patients ( 9 men, 3 women, age 45-69) after myocardial infarction (MI) that underwent percutaneous coronary intervention (PCI) on single coronary vessel (9 on LAD, 1 on left $\mathrm{Cx}$ and 2 on RCA) were used to assess the impact of the reperfusion on the AP and the surface potentials. It was hypothesized that due to the revascularization, regions with altered repolarization around the infarcted tissue will be reduced or disappear after the treatment. QRST integral maps before and after the intervention were computed from 32 ECG leads measured in the L32 lead set. Integral values in maps were corrected for the QT interval length if it varied by more than $5 \%$ between the measurements. Common realistic inhomogeneous torso and heart model geometry, the same as in the simulation study, were used 
Table 1. Errors (mean \pm standard deviation) of the inverse estimation of parameters of different types of lesions from 62 surface ECG leads using inhomogeneous or homogeneous torso model.

\begin{tabular}{llll}
\hline Parameter & Torso model & $\begin{array}{l}\text { Small \& medium } \\
\text { lesions }\end{array}$ & $\begin{array}{l}\text { Large transmural } \\
\text { lesions }\end{array}$ \\
\hline \multirow{2}{*}{ Localization error [mm] } & Inhomogeneous & $9 \pm 4$ & $17 \pm 14$ \\
& Homogeneous & $11 \pm 8$ & $16 \pm 15$ \\
\hline \multirow{2}{*}{ Dipole direction error [ $\left.{ }^{\circ}\right]$} & Inhomogeneous & $9 \pm 7$ & $14 \pm 4$ \\
& Homogeneous & $8 \pm 5$ & $17 \pm 7$ \\
\hline \multirow{2}{*}{ Dipole moment [\% of original] } & Inhomogeneous & $51 \pm 40$ & $221 \pm 206$ \\
& Homogeneous & $49 \pm 33$ & $163 \pm 123$ \\
\hline \multirow{2}{*}{ Relative difference of ED map [\%] } & Inhomogeneous & $9 \pm 4$ & $16 \pm 1$ \\
& Homogeneous & $12 \pm 2$ & $16 \pm 2$ \\
\hline
\end{tabular}

for all patients to find an ED representing the region with altered repolarization. In real measurements, possible positions of ED were defined at gravity centers of 28 anatomical segments of the analytical heart model.

\section{ECG mapping device}

Based on previous experience with BSP mapping devices (Rosik et al. 2002), a battery-powered ECG mapping system ProCardio-8 was developed to get high quality multi-channel ECG recordings necessary for BSP diagnostic interpretation and utilization as input data for inverse solutions. The system consists of a set of active electrodes, a data acquisition system and a hosting personal computer used for measurement control, processing of measured data and their analysis and interpretation. The data acquisition program permits to select the desired lead set, checks the electrode contacts and records ECG data. Signals from selected channels can be simultaneously monitored on the PC screen. Offline ECG processing includes signal filtering, body surface potential mapping and evaluation of the measured data using the above described method.

\section{Results}

\section{Simulated data}

AP changes simulated in three ventricular regions were projected into computed body surface potentials and were clearly visible in the corresponding areas of DI maps. Displayed differences increased with increasing lesion size and degree of AP shortening, except for transmural lesions where the differences were much smaller than in comparable non-transmural lesions. An example of simulated QRST integral maps and DI maps after AP was shortened by $20 \%$ in medium-sized subendocardial lesions A2, P2 and I2 is shown in Fig. 2 (top). AP shortening was projected as decrease of the QRST integrals mainly in the nearby left anterior, mid posterior and lower right anterior and posterior torso surface, respectively.

Minimum error of the inverse estimation of the ED localization was limited by the selection of predefined possible dipole positions. In our setup, distances between centers of the simulated lesions and the nearest possible positions of the ED were $1.7-7.3 \mathrm{~mm}$ (mean $5.5 \mathrm{~mm}$ ).

Results of the inverse ED estimation from 62 surface ECG leads with the use of homogeneous or inhomogeneous torso model are summarized in Table 1, showing mean errors of the ED localizations, dipole moment orientations and magnitudes, as well as mean relative differences between simulated DI maps and maps generated by the ED. Mean localization errors varied from $9 \pm 4 \mathrm{~mm}$ to $16 \pm 15$. For small and medium lesions, the maximum localization error reached $16 \mathrm{~mm}$ in the inhomogeneous torso and $23 \mathrm{~mm}$ in the homogeneous torso. Localization of large transmural lesions was not satisfactory and maximum error was $43 \mathrm{~mm}$. Directions of dipole moments were estimated with an error of $9 \pm 7$ to $17 \pm 7$ degrees, orientations matched with subepicardial or subendocardial character of the lesions and were nearly opposite. Relative errors of dipole moments were large, 


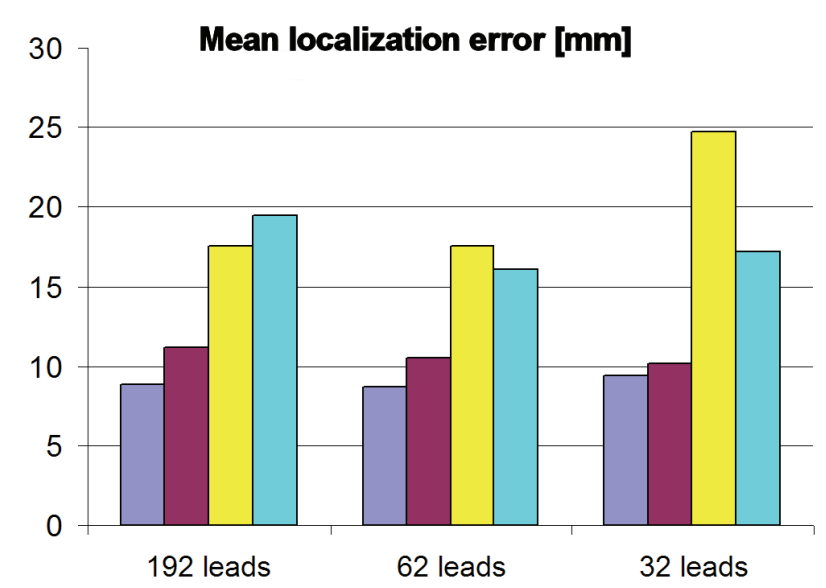

$\square$ small \& medium lesions - inhomogeneous torso

$\square$ small \& medium lesions - homogeneous torso

$\square$ large transmural lesions - inhomogeneous torso

$\square$ large transmural lesions - homogeneous torso

Fig. 3. Mean values of the localization error [mm] for small subendocardial and subepicardial lesions and for large transmural lesions when different lead sets and homogeneous or inhomogeneous torso models were used.

especially for transmural lesions. Relative rms deviations between original DI maps and maps computed from ED were from 9 to $16 \%$ and suggest that the dipole may be an adequate representation of small and medium lesions. Examples of three lesions with their corresponding summary dipolar sources and inversely calculated ED sources are illustrated in Fig. 2 (bottom).

Evaluation of the combined influence of the number of measured leads, size of the lesion and used torso model on the localization error is shown in Fig. 3. Localization of small lesions from 192 and 62 leads provided similarly good results; localization from 32 leads was less accurate for large lesions and inhomogeneous torso. For large lesions, the influence of the number of leads was higher. In most cases, results obtained with the use of homogeneous torso model were less accurate than with the inhomogeneous torso model.

The influence of the disturbances in QRST integrals is illustrated in Fig. 4. The mean localization error for all simulated lesions increased from $11 \mathrm{~mm}$ (when no disturbances were present) to $16 \mathrm{~mm}$ (when maximal disturbances N4 were added). There was no significant difference between the influence on computations using the inhomogeneous and homogeneous torso model. The influence of the disturbances on the dipole orientation and dipole moment was not significant. Relative difference between the original DI map and map computed from the ED source
Mean localization error [mm]

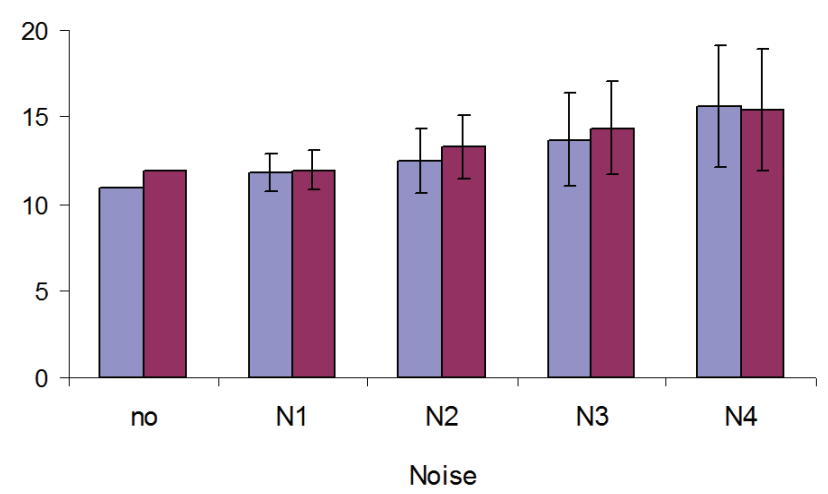

$\square$ inhomogeneous torso

$\square$ homogeneous torso

Fig. 4. Influence of random disturbances in QRST integrals on the mean localization error of the lesion with changed repolarization when using inhomogeneous and homogeneous torso model.

increased significantly from $12 \%$ to $32 \%$ (with no and N4 disturbances, respectively).

Measured ECG data

From the 12 tested MI patients, the PCI was successful in 11 patients. In one patient where the PCI failed, there were very small changes in the surface potentials and correspondingly small was the estimated dipole moment of the ED source.

In patients after successful PCI, the changes in QRST integral maps were noticeable. In 8 of them the measured DI maps could be reasonably represented by maps generated by single current dipole. In 6 cases the relative rms error between the measured DI maps and maps generated by the ED was $<35 \%$, in further 2 patients it was $<56 \%$. In the remaining 3 patients the rms error of the DI map approximation was higher than $56 \%$ and they were excluded from further analysis because single dipole was probably not the appropriate model for these cases. The QT interval was linearly corrected to compensate for the changed heart rate between the measurements in 6 of the 8 analyzed patients.

Despite the use of a common torso model, in 7 of 8 analyzed patients the positions of estimated ED matched with the region supplied by the treated vessel and the EDs were located near the anterior or posterolateral wall of the left ventricle. Dipole moments were directed inward the ventricle and suggested changes near the endocardial wall. In the remaining one patient after PCI on RCA the ED was probably incorrectly located in mid anterior left ventricular wall. 


\section{Discussion}

Our previous attempts to detect ischemic regions with changed repolarization by using departure integral maps (Filipová et al. 2003) showed that changes in BSP are small when compared with normal inter-individual fluctuations and can hardly be detected by departures from mean normal integral maps. However, relative rms differences between simulated normal and changed QRST integral maps were $20-45 \% \mathrm{rms}$, correlations $0.45-0.99$. These were greater than observed total intraindividual variability in maps in healthy subjects (5-20\% rms, correlations $>0.98$ ) what, in principle, allows their identification e.g. by the method proposed in this study.

Only the AP duration was changed in simulations of ischemic lesions presented in this study. Simultaneous changes of AP magnitudes were also tested with similar results. Changing only AP duration should not invade the results of the simulations because of the additive effect of both AP shortening and magnitude on the QRST integrals.

Our simulation showed that the use of the method for identification of larger transmural lesions might not be appropriate. In such cases the single dipole model is not adequate and its localization may not be in correspondence with the real lesion position. Moreover, transmural lesions produced smaller differences in the DI maps and their identification is more difficult. This fact should be taken into account also when evaluating real data.

Limitation of the simulation study is the use of isotropic ventricular activation model with analytical geometry and without atria. AP shapes and durations in the myocardium elements were defined a priori and possible electrotonic coupling was not included. No individual torso geometry was available when testing the method on real patients and common model geometry had to be used. As shown in our previous study (Tyšler et al. 2005) use of individual geometry should improve the accuracy of the lesion localization.

Simulated ED localization from 32 ECG leads provided slightly worse results than from 192 or 62 leads. Hence more than 32 leads should be used in future measurements, if possible. Therefore, the use of the highresolution mapping system with sufficient number of measuring channels seems to be crucial for practical implementation of the proposed method and its experimental testing.

When evaluating real data, the ED moment directions in several cases were not normal to the particular heart wall and expected lesion border. This may reflect the specific form of the affected area or anisotropy in the real myocardium that were not considered in the simulations.

Despite the limitations of the study, the presented results of the simulations and testing on real data suggest that local repolarization changes in different heart regions are clearly manifested in changed QRST integral maps and that their approximate site in the ventricular myocardium can be identified using a single equivalent dipole model. Its orientation can help to characterize the subepicardial or subendocardial character of the lesion. In 7 of 8 evaluated MI patients after successful PCI the presented method could localize the ED near the expected revascularized region. However, in 3 other patients the repolarization changes could not be sufficiently represented by single dipole and the patients had to be excluded from the evaluation. Therefore, a further study would be desirable to examine the applicability of the method on a larger group of patients, possibly with known individual torso geometry.

\section{Acknowledgments}

This work was supported by grants MSM 6840770012 from the Ministry of Education, Youth and Sports of the Czech Republic, 2/7092/27 from the VEGA grant agency and APVV- 51- 059005 from the APVV agency of the Slovak Ministry of Education.

\section{References}

ABILDSKOV JA, EVANS AK, LUX RL, BURGESS MJ: Direct evidence relating QRST deflection area and ventricular recovery properties. Circulation 60 (Suppl II): II-110, 1979.

ABILDSKOV JA, EVANS AK, LUX RL, BURGESS MJ: Ventricular recovery properties and QRST deflection area in cardiac electrogram. Am J Physiol 239: H227-H231, 1980. 
ANTZELEVITCH C., SHIMIZU W, YAN GX, SICOURI S, WEISEENBURGER J, NESTERENKO VV, BURASNIKOV A, Di DIEGO JM, SAFFITZ JE, THOMAS GP: The M cell. Its contribution to the ECG and to normal and abnormal electrical function of the heart. J Cardiovasc Electrophysiol 10: 1124-1152, 1999.

ANTZELEVITCH C: Transmural dispersion of repolarization and the T vawe. Cardiovasc Res 50: 426-431, 2001.

De AMBOGGI L, MUSSO E, TACCARDI B: Body surface mapping. In Comprehensive Electrocardiology. PW MACFARLANE, TDW LAWRIE (eds), Pergamon Press, New York, 1989, pp 1015-1049.

FILIPOVÁ S, TYŠLER M, TURZOVÁ M, ROSIK V: Reference ECG-mapping etalons improve the diagnostic accuracy of myocardial ischemia according to departure isointegral surface maps Int J Bioelectromagnet 5: 369-370, 2003.

GABOR D, NELSON CV: Determination of the resultant dipole of the heart from measurements on the body surface. $J$ Appl Physiol 24: 413-416, 1954.

GARDNER MJ, MONTAGUE TJ, HORACEK BM, CAMERON DA, FLEMINGTON CS, SMITH ER: Vulnerability to ventricular dysrhytmia: assessment by body surface mapping. Circulation 64 (Suppl IV): IV-328, 1981.

GESELOWITZ DB: The concept of an equivalent cardiac generator. In: Biomedical Science Instrumentation 1. F ALT (ed.), Plenum, New York, 1963, pp 325-330.

GULRAJANI RM, ROBERGE FA, SAVARD P: The inverse problem of elelectrocardiography. In: Comprehensive Electrocardiology. PW MACFARLANE, TDV LAWRIE (eds), Pergamon Press, 1989, pp. 237-288.

HOEKEMA R, UIJEN GJH, VAN OOSTEROM A: On selecting a body surface mapping procedure. $J$ Electrocard 32: 93-101, 1999.

HORÁČEK BM, WARREN JW, PENNEY CJ, MACLEOD RS, TITLE LM, GARDNER MJ, FELDMAN CL: Optimal electrocardiographic leads for detecting acute myocardial ischemia. J Electrocard 34 (Suppl.): 97-111, 2001.

KORNREICH F, RAUTAHARJU PM, WARREN JW, HORACEK BM, DRAMAIX M: Effective extraction of diagnostic ECG waveform information using orthonormal basis functions derived from body surface potential maps. J Electrocard 18: 341-350, 1985.

KOZLÍKOVÁ K, HULÍN I, MURÍN J, BULAS J: Quantitative evaluation of mean repolarization changes: a long-term follow-up after myocardial infarction. Biomedizin Technik 42 (Suppl. 1): 128-131, 1997.

LI GR, FENG J, YUE L, CARRIER M: Transmural heterogeneity of action potentials and Ito1 in myocytes isolated from the human right ventricle. Am J Physiol 275: H369 - H377, 1998.

LUKAS A, ANTZELEVITCH C: Differences in the electrophysiological response of canine ventricular epicardium and endocardium to ischemia. Role of transient outward current. Circulation 88: 2903-2915, 1993.

LUX RL, SMITH CR, WYATT RF, ABILDSKOV JA: Limited lead selection for estimation of body surface potential maps in electrocardiography. IEEE Biomed Eng BME-25: 270-276, 1978.

MALMIVUO J.: Solution of bioelectric and biomagnetic sources. In: XIV ICMBE and VII ICMP, ESPOO, 1985, pp. 73-76.

MIRVIS DM: Electrocardiography. A Physiological Approach. Mosby-Year Book, USA, 1993.

ROSIK V, TYŠLER M, JURKO S, RASO R, TURZOVÁ M: Cardio 7 - portable system for high-resolution ECG mapping. Studies Health Technol Informatics 90: 41-46, 2002.

SAVARD P, ROBERGE FA, PERRY J, NADEAU RA: Representation of cardiac electric activity by a moving dipole for normal and ectopic beats in the intact dog. Circ Res 46: 415-425, 1980.

SIPPENGROENEWEGEN A, SPEKHORST H, VAN HEMEL NM, KINGMA JH, HAUER RNW, GRIMBERGEN CA, JANSE MJ, DUNNING AJ: Value of body surface mapping in localization of the site of origin of ventricular tachycardia in patients with previous myocardial infarction. $J$ Am Coll Cardiol 24: 1708-1724, 1994.

SZATHMÁRY V, OSVALD R: An interactive computer model of propagated activation with analytically defined geometry of ventricles. Comput Biomed Res 27: 27-38, 1998.

SZATHMÁRY V, RUTTKAY-NEDECKÝ I: Effect of different sources of ventricular repolarization heterogeneity on the resultant cardiac vectors. A model study. Health Technol Informatics 90: 88-92, 2002. 
TRUDEL MC, DUBÉ B, POTSE M, GULRAJANI RM, LEON LJ: Simulation of QRST integral maps with a membrane-based computer heart model employing parallel processing, IEEE Biomed Engin 51: 1319-1329, 2004.

TYŠLER M, TURZOVÁ M, ŠVEHLÍKOVÁ J, HEBLÁKOVÁ E, FILIPOVÁ S: Noninvasive detection of ischemic regions in the heart. IFMBE Proc 11: 2207, 2005.

TYŠLER M, SZATHMÁRY V, TURZOVÁ M: Model study of assessment of local heart repolarization changes by several ECG methods. Int J Bioelectromagnet 5: 252-253, 2003.

YAN GX, SHIMIZU W, ANTZELEVICH CH: Characteristics and distribution of M cells in anterially perfused canine left ventricular wedge preparations. Circulation 98: 1921-1927, 1998.

\section{Corresponding author}

Peter Kneppo, Faculty of Biomedical Engineering, Czech Technical University, Nám. Sítná 3105, 27201 Kladno, Czech Republic. E-mail: kneppo@fbmi.cvut.cz. 